\title{
Strategy of Sustainable Use of Biological Resources
}

\section{GUO ZHONGWEI}

Institute of Zoology. Academia Sinica, 100080 Beijing, PRC.

(Received December, 16. 1994)

\section{ABSTRACT}

In this paper, the theory of Multi-Objective Optimal Sustainable Yield is developed based on the theory of optimal sustainable yield, which makes multiple objectives about biology, environment. economy and society optimization. The Strategy of Satisfied Sustainable Yield is an approach to determine a satisfied yield close to optimum yield. resulted from the principle of Multiobjective optimal sustainable yield, and a managing strategy to aid satisfied yield. The approach provides bases for the management of biological resource. The Strategy of Satisfied Sustainable Yield for Waterhyacinth, as a case study, is discussed.

Key words biological resources, multi-objective optimal sustainable yield. strategy of satisfied sustainable yield

\section{Introduction}

The drastic growth of the world population is obviously one of the most noteworthy aspects of modern history. The escalating human needs for food, fibre, shelter and health have frequently led to a short-sighted approach and unsustainable technologies for exploitation of biological resources. This has resulted in rapid depletion of biological resources. Awareness of human impact on the biodiversity of conservation has grown rapidly over the past a decade and the ideas of conservation of biodiversity and sustainable uses of biological resources have been accepted extensively. The sustainable use of biological resources may be understood as gaining profits from biological resources within the carrying capacity of supporting ecosystem, using the cultural and organizational capabilities of society. However. it is easy to recognize the good idea, but it is difficult to put the good idea into practice, for the complexities of biological system.

The theories of optimal sustainable yield are mostly based on the principle of optimal control. Applications of optimal control theory to ecological problems appeared in the late 1960s (Watt, 1968: Goh, 1969/1970; Becker. 1970: Clark, 1971). In 1970s, (Clark. 1976: Conway, 1977; Wickwire, 1977) reviewed the applications of optimal control theory in resource management and the control of epidemics.

Since there are many differences between biological system and physical system and the theory of optimal control is developed based on physical system, it is difficult that theoretical optimum results are carried out in biological system. In practice, when the problems of optimal control for biological system are studied, the "satisfied results" should be determined based on biological characteristics. 


\section{Strategy of sustainable use of biological resources}

2. 1 Multi-objective optimal sustainable yield

The values of biological resources are multiple for human. The forest, for example, is not only a resource of wood but also a resource of environment, which is able to release $\mathrm{O}_{2}$, absorb $\mathrm{CO}_{2}$, keep moisture and regulate climate. This multiplicity of value results in the diversity of benefits gained from biological resource. From forest, human can gain the benefits of both economy and environment. Obviously, if a certain of use of biological resource is carried out by harvesting, the benefits provided by alive organism should be considered as factors controlling the quantity of yield. "Sustainable use" should include multiple values of use for biological resources.

Moreover, since organism, which is used as a biological resource, is a part of ecosystem, its existence as well as quantity and distribution is important to ecosystem in different degree, as should be considered as the controlling factors of yield, too.

The quantity of yield only depends on the economic benefit gained from biological resource and the capacity of population recovering in the traditional theory of sustainable yield (Holt et al. . 1978), neglecting the affections to environmental and social benefits caused by yield. To overcome its deficiencies Multi-Objective Optimal Sustainable Yield is developed. So-called Multi-objective means that the controlling objectives are the multiple and controlling aim are to make the multiple objectives about biology, environment. economy and society optimization in the sustainable use of biological resource.

The theory of Multi-Objective Optimal Sustainable Yield is based on the principle of optimal control. When the yields are determined, the biological, environmental, economic and social benefits gained from biological resource as well as the capacity of population recovering will be considered and sometime give particular emphasis on some factors in a specific problem. The main content of the theory is that optimal yield is determined by the principle of optimal control, depending on the dynamics of quantity of population based on environmental carrying capacity and the intrinsic growth rate of population as well as the dynamics of environmental, economic and social factors caused by the quantity of population and determining a set of data as terminal standards indicating the environmental, economic and social effects hoped.

Let $B$ be the density of biological resource population, $F c_{\mathrm{i}}(\mathrm{i}=1,2, \ldots, \mathrm{n})$ be a set of environmental, economic and social variates. Let $\theta_{\mathrm{j}}\left(F c_{\mathrm{i}}\right) \quad(\mathrm{i}=1,2, \ldots, \mathrm{n} ; \mathrm{j}=1,2, \ldots ., \mathrm{m})$ be a set of functions indicating the affections of population to environmental, economic and social factors and $u(t)$ be the rate of harvest. Let $b, F_{c_{\mathrm{if}}}(\mathrm{i}=1,2, \ldots . \mathrm{n})$ and $u_{\max }^{\prime}$ be positive constants. The planning period is $\left[0, t_{\mathrm{f}}\right]$, with the nonextinction constraint $B$ $\left(t_{\mathrm{f}}\right) \geqslant b$, benefits constraint $F_{c_{\mathrm{i}}}\left(t_{\mathrm{f}}\right) \geqslant F c_{\mathrm{if}}$ and quota constraint $0 \leqslant u \leqslant u_{\max }$. The mathematical description of Multi-objective optimal sustainable yield can be expressed as follows:

$$
\begin{array}{ll}
\text { System: } & \mathrm{d} B / \mathrm{d} t=r B(1-B / K)-u \\
& \mathrm{~d} F c_{\mathrm{i}} / \mathrm{d} t=\theta_{\mathrm{j}}\left(F c_{\mathrm{i}}\right) \quad \mathrm{i}=1,2, \ldots, \mathrm{n} ; \mathrm{j}=1,2, \ldots \mathrm{m} ; \\
\text { Initially: } & B(0)=B, F c_{\mathrm{i}}(0)=F c_{\mathrm{i} 0} ; \\
\text { Terminally: } & F c_{\mathrm{i}}\left(t_{\mathrm{f}}\right) \geqslant F c_{\mathrm{if}}, B\left(t_{\mathrm{f}}\right) \geqslant b ; \\
\text { Constraints: } & 0 \leqslant u \leqslant u_{\max } ;
\end{array}
$$

$\cdot 80 \cdot$ 


$$
\text { Objective: } \min -G\left[t_{\mathrm{f}}, F_{c_{\mathrm{i}}}\left(t_{\mathrm{f}}\right)\right]-\operatorname{t}_{0}^{t_{f}} L(B, u) \mathrm{d} t .
$$

Where $r$ and $k$ are positive constants. $G$ and $L$ denote scale functions. In order to use the standard continuous-time optimal control problem directly the maximization problem is converted itno a minimization problem.

\section{2 Strategy of satisfied sustainable yield}

The essence of optimal control is to turn complex reality into a simpler mathematical framework and to optimize it according to some fixed rules. Though it is a good method for well-defined physical system, it is not suitable for biological system. In general, the mechanisms of biological system are more complex than those of physical system so that the optimum results can not be easily accepted. In a specific problem, it is available to determine a "satisfied result" close to optimum result and easily carried out. Here an approach, called the Strategy of Satisfied Sustainable Yield (SSSY), based on Multi-Objective Optimal Sustainable Yield is developed to manage these ill-structured systems.

SSSY is a biological resource managing method aimed at determining an actual yield easily accepted and close to the optimum result of Multi-Objective Optimal Sustainable Yield, by means of regulating some factors related to yield.

The regulations include two aspects:

\section{2. 1 The regulation of the rate and interval of harvest}

The theoretical result of optimal control provides a strict control trajectory being suitable to physical system. However, if this strategy of management is carried out in biological system, it may mean that a smaller quantity of yièld is done in a shorter interval of time (e. g. , $0.5 \mathrm{~kg}$ of biomass $/ \mathrm{m}^{2}$ per day). Obviously, that way of yield is difficult to be executed and economic benefit is lower.

To overcome above problem, on the conditions that other objectives keep optimization and the difference between actual yield and optimum yield is reasonable, we expend the interval of time for yield and regulate the quantity of harvesting relevantly so that the interval and the quantity of harvesting are easily accepted. That expansion should deperid on the growth period of population and meet the needs of other factors for the density of population. In this way, we have a "satisfied yield".

\section{2. 2 The regulation of structure}

It is usually important for sustainable yield and sometime for the optimization of other factors that the structures of age, sex and distribution of population are suitable. The aim of structure regulating is to work out a strategy of managing culture and yield to make the structures of population meeting the needs of satisfied sustainable yield as well as environment, economy and society, especially after harvesting. The regulation of structure usually depends on the growing period of population and the varieties of density of population caused by harvesting and supplement each other with the regulation of the rate and interval of harvest.

By above approaches, we have a satisfied yield colse to optimum yield and a strategy of management to aid satisfied yield, i. e., the Strategy of Satisfied Sustainable Yield. SSSY provides an effective approach for the management of biological resource.

\section{- 3 Case study}


In Suzhou, the Province of Jiangsu, China, waterhyacinth is used for purifying polluted water in outer city moat. Waterhyacinths and organic contaminators agglutinated on plant are removed out of river by yielding. In this way, the outer city moat is purified and Waterhyacinths processed are green fodder fed by fish. duck, pig and cattle, gaining notable environmental and economic benefits. Here, Waterhyacinth is a biological resource holding multiple uses.

3. 1 Model of multi-objective optimal sustainable yield for waterhyacinth

Waterhyacinth holds multiple functions of purifying polluted water, which depend on its density. The reasonable density of Waterhyacinth should be maintained to decrease contaminators in river and a fixed quantity of Waterhyacinths is harvested to remove contaminators out of river and be green fodder.

The dynamic equation of density of Waterhyacinths is. given by:

$$
\mathrm{d} B / \mathrm{d} t=r B(1-B / K)-u
$$

Here, $r, K$ and $u$ are Waterhyacinth's intrinsic growth rate, its environment carry capacity and its rate of harvest, respectively.

The yield is based on the contaminator's content in river, which relates to the functions of treating contaminators of Waterhyacinth (Yan Jingsong, 1986). Given: The area of Waterhyacinth planting is $A$, in a section of river, when flow $Q$, containing concentration $C i$, enters the area in unit time (day), contaminator's concentration is $C o$ in outlet, then dynamic equation of contaminator's concentration in river is given by:

$$
\mathrm{d} C o / \mathrm{d} t=C i-B^{\prime} A E i / Q
$$

Here, $E i$ is the quantity of contaminator purified by Waterhyacinth in unit area per day.

Since contaminators's concentration in Waterhyacinths affects the effect of purification, the dynamic equation of concentration in Waterhyacinths is given by:

$$
\mathrm{d} C p / \mathrm{d} t=\kappa_{\mathrm{u}} C i-\rho C p
$$

Here, $C p$ is the concentration of contaminators in Waterhyacinth $(\mathbf{k g}) ; \kappa_{\mathrm{u}}$ is the sorption of contaminators from water $\left(\right.$ day $\left.^{-1}\right) ; \rho$ is desorption rate $\left(\right.$ day $\left.^{-1}\right)$.

Here, control system includes Waterhyacinth and contaminator. The control aim of the system is to gain maximum rate of harvest of Waterhyacinths $(u)$ on the condition that the concentration of contaminators at outlet $(C o)$ and in Waterhyacinth $(C p)$ satisfy standard hoped, respectively, i. e., $C o\left(t_{\mathrm{f}}\right) \leqslant C_{\mathrm{s}}$ and $C p\left(t_{\mathrm{f}}\right) \leqslant C p_{\mathrm{s}}$. The quota constraint is $0 \leqslant u \leqslant u_{\max }$. The mathematical description of Multi-objective optimal sustainable yield for Waterhyacinth can be expressed as follows:

$$
\begin{array}{ll}
\text { System: } & \mathrm{d} B / \mathrm{d} t=r B(1-B / K)-u \\
& \mathrm{~d} C o / \mathrm{d} t=C i-B A E i / Q \\
& \mathrm{~d} C p / \mathrm{d} t=\kappa_{\mathrm{u}} C i-\rho C p \\
\text { Initially: } & C o(0)=C_{0} ; B(0)=B_{0} ; C p(0)=C_{p_{0}} \\
\text { Terminally: } & C o\left(t_{\mathrm{f}}\right) \leqslant C_{\mathrm{s}} ; C p\left(t_{\mathrm{f}}\right) \leqslant C p_{\mathrm{s}} ; \\
\text { Constraints: } & 0 \leqslant u \leqslant u_{\max } ; \\
\text { Objective: } & J=\min \alpha C_{o}+\beta C p-{ }_{t_{0}}^{t_{f}} \int u \mathrm{~d} t .
\end{array}
$$

Here, $\alpha$ and $\beta$ are weight factors satisfying for $\alpha, \beta \in[0,1]$. The values of $\alpha$ and $\beta$ can be chosen to determine the purifying effect and the concentration in Waterhyacinths.

3. 2 Optimum yield for waterhyacinth

By the Maximum Principle, solving above optimal problem, we have the optimal 
singular control, i. e., optimum rate of harvest, $u^{*}$ and the corresponding optimal density of Waterhyacinth, $B^{*}$. Then, the optimal control is divided into three partitions:

(a) when $B>B^{*}, u=u_{\max }$, till $B=B^{*}$;

(b) when $B=B^{*}$, optimal singular control is carried out, $u=u^{*}$, till $B<B^{*}$;

(c) when $B<B^{*}, u=0$.

Then, we know the optimal control for Waterhyacinths is a Bang-Singular-Bang control. Once $B=B^{*}$ is satisfied, the control only moves forth and back during (b) and (c).

3. 3 The strategy of satisfied sustainable yield for waterhyacinth

The channel in which Waterhyacinth grow is length about $663 \mathrm{~m}$, which is control object. In the upper reaches of the channel, the concentration of COD is $26.29 \mathrm{mgO}_{2} / \mathrm{L}$. The area of Waterhyacinth's growing is about $1000 \mathrm{~m}^{2}$ to make COD in lower reaches, Co, below $24.9 \mathrm{mgO}_{2} / \mathrm{L}$ and the COD in Waterhyacinth, $C p$, should be below $19 \mathrm{mgO}_{2} /$ $\mathbf{k g}$ (wet). Applying Bang-Singular-Bang control to the control object and simulated by computer (where $E i \approx 0.06 \mathrm{mgO}_{2} / \mathrm{kg}$ (wet). day, $\kappa_{\mathrm{u}} \approx 0.018 \mathrm{mgO}_{2} / \mathrm{kg}$ (wet). day; $P \approx$ $0.003, \alpha \approx 0.2$ and $\beta=0.06$ ) the result shows (Fig. 1): the optimal density of Waterhyacinths is $14.6 \mathrm{~kg}$ (wet) $/ \mathrm{m}^{2}$, optimal rate of harvest is $0.5 \mathrm{~kg}$ (wet) $/ \mathrm{m}^{2}$. When $t=16$

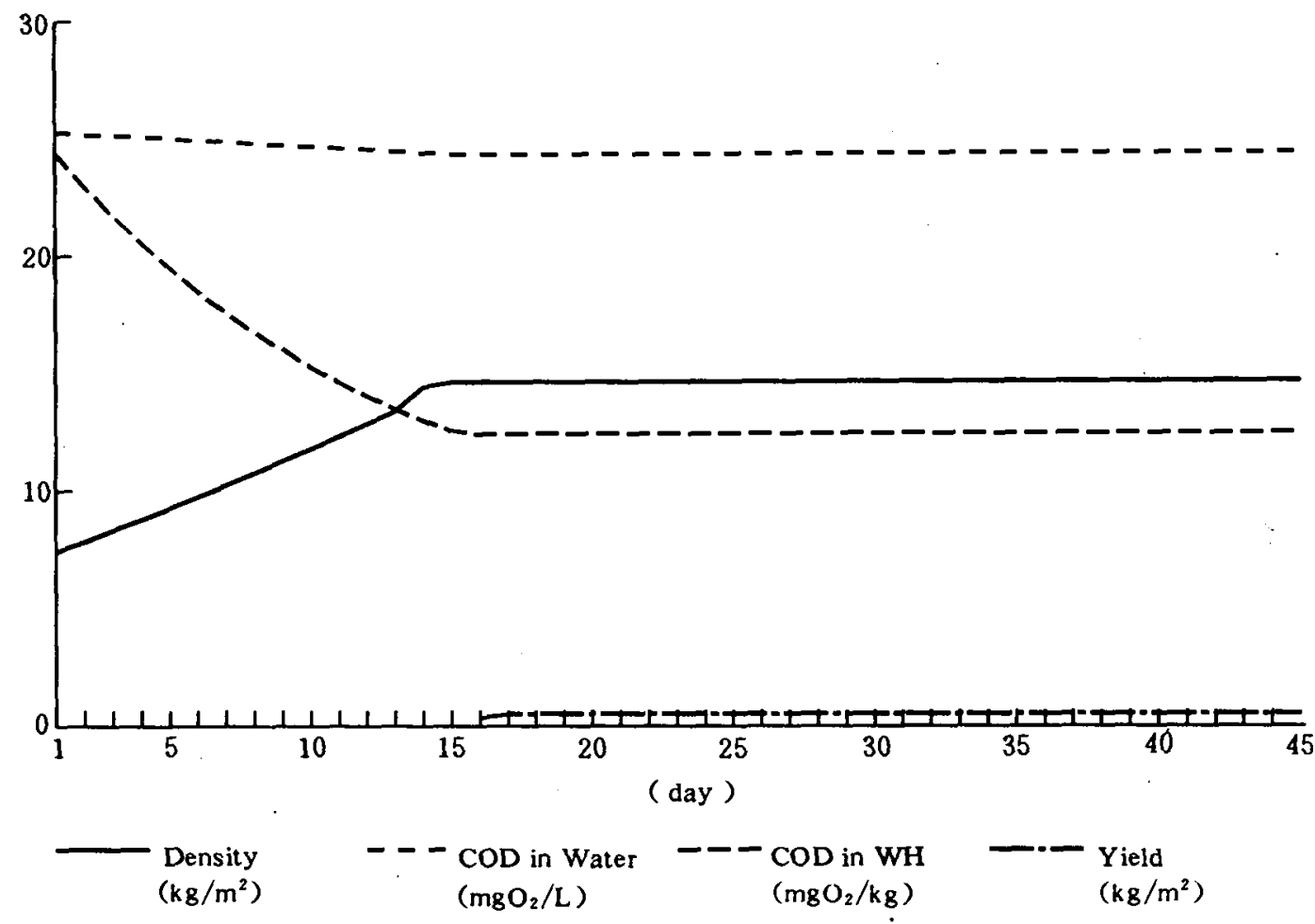

Fig. 1 The optimum result of multi-objectives sustainable yield for waterhyacinth.

Note: Original data are collected from the outer city moat of Suzhou. Province of Jiangsu, in April May.

(days), density is over $14.6 \mathrm{~kg}$ (wet) $/ \mathrm{m}^{2}$; yield begins with $0.33 \mathrm{~kg}$ (wet) $/ \mathrm{m}^{2}$. From $t=$ 17 (days), density maintains $14.6 \mathrm{~kg}$ (wet) $/ \mathrm{m}^{2}$ and yield maintains $0.5 \mathrm{~kg}$ (wet) $/ \mathrm{m}^{2}$ per 
day, the $C o$ is $24.3 \mathrm{mgO}_{2} / \mathrm{L}$ and $C p$ is $12.43 \mathrm{mgO}_{2} / \mathrm{kg}$ (wet), satisfying standards hoped. The system is optimal.

Since it is difficult that the rate of harvest is only $0.5 \mathrm{~kg}$ (wet) $/ \mathrm{m}^{2}$ per day and the yield should be as well-disturbed as possible in whole area, which is important to purifying polluted water. On the basis of the optimum yield, we make out the Strategy of Satisfied Sustainable Yield for Waterhyacinth.

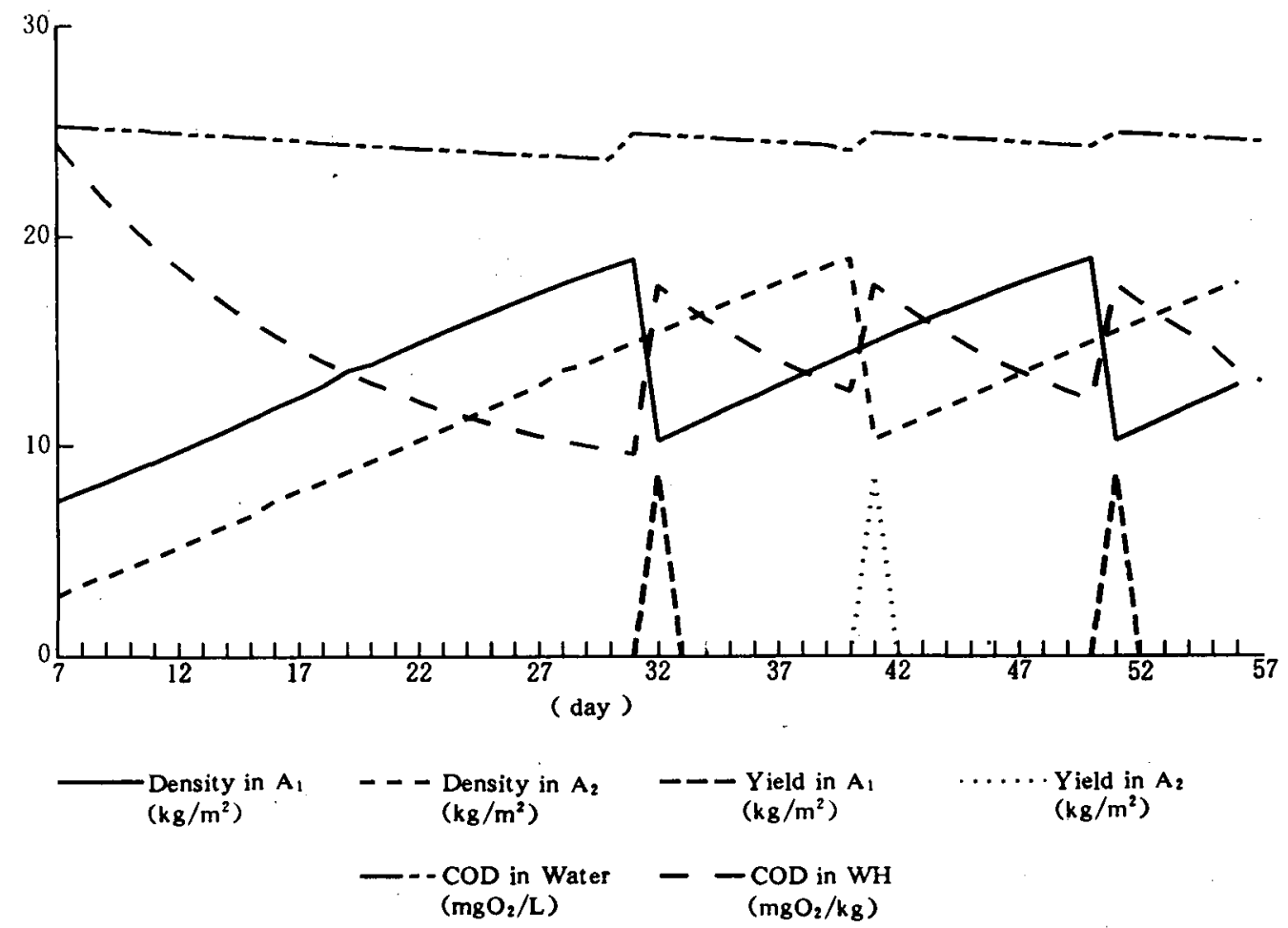

Fig. 2 The satisfied result of the strategy of satisfied sustainable yield for waterhyacinth.

Note: Original data are collected from the outer city moat of Suzhou. Province of Jiangsu. in April $\sim$ May.

When density is $10.28 \mathrm{~kg}$ (wet) $/ \mathrm{m}^{2}$ in seventh day, Co and $C p$ have satisfied purifying standards and the difference between optimum density, $14.6 \mathrm{~kg}$ (wet) $/ \mathrm{m}^{2}$, and $10.28 \mathrm{~kg}$ (wet) $/ \mathrm{m}^{2}$ is $4.32 \mathrm{~kg}$ (wet) $/ \mathrm{m}^{2}$, then, it is feasible that let the density of Waterhyacinth waves about optimum density within the range of \pm 4.32 . And, since it takes nineteen days that the density of Waterhyacinth rises from $10.28 \mathrm{~kg} / \mathrm{m}^{2}(14.6-4.32)$ to $18.92 \mathrm{~kg}$ (wet) $/ \mathrm{m}^{2}(14.6+4.32)$, by the principle of rotational planting and rotational harvesting, the planting area is be divided into two same sections $\left(A_{1}\right.$ and $A_{2}$ ) perpendicular to the direction of river's flowing. In the two sections, Waterhyacinths are planted and harvested at different time. The interval of time of Waterhyacinth's planting and harvesting should be nine or ten days between the two sections. When density is 
$18.92 \mathrm{~kg}$ (wet) $/ \mathrm{m}^{2}$ ir. $\mathrm{A}_{1}$, Waterhyacinths are harvested in it by the yield of $8.64 \mathrm{~kg}$ (wet) $/ \mathrm{m}^{2}$; the density in $\mathrm{A}_{1}$ decreases to $10.28 \mathrm{~kg}$ (wet) $/ \mathrm{m}^{2}$. After nine days, when the density in $A_{2}$ is $18.92 \mathrm{~kg}$ (wet) $/ \mathrm{m}^{2}$. Waterhyacinths are also harvested in it by the yield of $8.64 \mathrm{~kg}$ (wet) $/ \mathrm{m}^{2}$; the density in $A_{2}$ also decreases to $10.28 \mathrm{~kg}$ (wet) $/ \mathrm{m}^{2}$. After ten days, the yield is car:ied out in $A_{1}$ again. In this way. Waterhyacinths in $A_{1}$ and $A_{2}$ are harvested at intervals of nineteen days, respectively, in which harvesting can be carried out by more quantity and at longer interval than even. moreover since harvesting is carried out stage by stage and section by section, the density and the structure of distribution are maintained in comparative well-disturbed in a section enough to make $C o$ and $C p$ below standards hoped (Fig. 2). Then, we adopt the managing strategy as the Strategy of Satisfied Sustainable Yield for Waterhyacinth.

\section{Conclusion}

The theory of Multi-Objective Optimal Sustainable Yield provides an effective approach to make the hiological, environmental, economic and social objective optimization in the use of biological resources. It is a progress changing singular-objective use of biological resource to multi-objective, which is helpful for human getting a deeper understanding on the use of biological resource.

The whole procedure of the Strategy of Satisfied Sustainable Yield is an intelligence-aided decision support process, which makes out an available scheme based on the result of Multi-Objective Optimal Sustainable Yield, 'according to expert' s knowledge about actual biologicel system. Obviously, that is an effective way to resolve the problem about how to apply the principles and the methods of mathematics to biological system.

\section{APPENDIX}

In this paper, optimal control problem is solved by the Maximum Principle (Xie Xueshu, 1986). Let $\lambda(t)_{i}(i=1,2,3)$ is costate vector, then Hamiltonian function is defined:

$H(B, C o, C p, u, \lambda)=-u+\lambda_{1}(r B(1-B / K)-u)+\lambda_{2}(C i-(B A E i) / Q)+\lambda_{3}\left(\kappa_{u} C i-\right.$ $\rho C p)$

According to: $\mathrm{d} \lambda / \mathrm{d} t==-\partial H / \partial x, \quad\left(x=[B, C o, C p]^{t}\right)$

Costate equations are: $\mathrm{d} \lambda_{1} / \mathrm{d} t=-\lambda_{1}(r-2 B r / K)+\lambda_{2} A E i / Q ; \mathrm{d} \lambda_{2} / \mathrm{d} t=0 ; \mathrm{d} \lambda_{3} / \mathrm{d} t=\lambda_{3} \rho$

Boundary conditions (Xie Xueshu, 1985) are: $\lambda_{2}\left(t_{f}\right)=\alpha ; \lambda_{3}\left(t_{f}\right)=\beta$

Based on Maximum F'rinciple, optimal yield $\mathrm{u}(\mathrm{t})$ should make $H(B, C o, C p, u, l) \mathrm{min}$ imum, or

$$
\begin{aligned}
H(B, C o, C p, \text { u. } \lambda)= & -u+\lambda_{1}(r B(1-B / K)-u)+\lambda_{2}(C i-(B A E i) / Q)+\lambda_{3}\left(\kappa_{u} C i\right. \\
& -\rho C p)
\end{aligned}
$$

minimum. Then, $H$ minimizing results from $-u\left(1+\lambda_{1}\right)$ minimizing. By calculating, there are three possibilities: $=0$.

$u=0 \quad$ only if $1+\lambda_{1}<0 ; \quad u=u_{\max } \quad$ only if $1+\lambda_{1}>0 ; \quad 0<u<u_{\max }$ only if $1+\lambda_{1}$

If very few points satisfy $1+\lambda_{1}=0$ in interval $t_{0} \leqslant t \leqslant t_{\mathrm{f}}$, optimal rate of harvest $u(t)$ is a 
Bang-Bang control (Xie Xueshu, 1986).

Because $H$ is linear function of control vector $u$, optimal rate of harvest $u^{*}$ may be singular, or there is at least a sub- interval $\left[t_{1}, t_{2}\right] \in\left[t_{0}, t_{f}\right]$ satisfying below relations:

$$
\begin{aligned}
H_{\mathrm{u}}=1+\lambda_{1}= & 0 ; \mathrm{d}\left(H_{\mathrm{u}}\right) / \mathrm{d} t=-\lambda_{1}(r-2 B r / K)+\lambda_{2} A E i / Q=0 ; \\
\mathrm{d}^{2}\left(H_{\mathrm{u}}\right) / \mathrm{d} t^{2}= & \lambda_{1}\left((r-2 B r / K)+\lambda_{2}(A E i) / Q\right)\left((r-2 B r / K)+\lambda_{2}(A E i) / Q\right)+2 \lambda_{1} r / \\
& K=0
\end{aligned}
$$

and.

$$
\begin{aligned}
\partial\left[\mathrm{d}^{2}\left(H_{\mathrm{u}}\right) / \mathrm{d} t^{2}\right] / \partial u= & \lambda_{1}\left(-4 B_{\mathrm{t}} r^{2} / K+8 B_{\mathrm{t}} r^{2} / K^{2}+4 \lambda_{2} B_{\mathrm{t}} \mathrm{r} E i / Q\right)-\lambda_{1 \mathrm{t}}((r-2 B r / K) \\
& \left.+\lambda_{2} A E i / Q\right)\left((r-2 B r / K)+\lambda_{2} A E i / Q\right)+2 \lambda_{1 \mathrm{t}}(r / K)<0
\end{aligned}
$$

here, $B_{\mathrm{t}}$ and $\lambda_{\mathrm{t}}$ denote $\mathrm{d} B / \mathrm{d} t$ and $\mathrm{d} \lambda / \mathrm{d} t$. Then, the singular solution $\left(B^{*}, u^{*}\right)$ satisfies Theorem 5 (Halfon, 1979). Solving above eqs., we have the optimal singular control $u^{*}$ and the corresponding optimal trajectory $B^{*}$. Then, the optimal control is divided into three partitions:

(a) when $B>B^{*}, u=u_{\max }$, till $B=B^{*}$, phase point $(B, C o, C p$ ) reaches on singular arc; (b) when $B=B^{*}$, optimal singular control is carried out, $u=u^{*}$, phase point $(B, C o, C p)$ moves along specific singular arc till $B<B^{*} ;(c)$ When $B<B^{*}, u=0$.

\section{REFERENCES}

Becker N G, 1970. Control of a pest population. Biometrics, 26: 365 375

Bell D J. D H Jacobson, 1975. Singular Optimal Control Problem. New York: Academic Press

Bellman R E, 1957. Dynamic Programming. New Jersey: Princeton Univ. Press

Clark C W, 1971. Economically optimal policies for the utilization of biological renewable resources. Math. Biosci., 12: 245 260

Clark C W. 1976. Mathematical Bioeconomics: The Optimal Management of Renewable resources. New York

Conway G R, 1977. Mathematical models in applied ecology. Nature, 269:291 297

Goh B S, 1966. Necessary conditions for singular extremals involving multiple control variables. SIAMJ. Control, 4: 715 731

Goh B S, 1969/1970. Optimal control of a fish resource. Malayan Sci., 5: 65 70

Halfon E. 1979. Theoretical Systems Ecology. New York: Academic Press

Holt S J. L M Talbot. 1978. New principles for the conservation of wild living resources. Wildl. Monogr., 59:1 33

Kelley H J, R E Kopp. H G Moyer, 1967. Singular extremals. In: G Leitmann (ed.) Topics in Optimization" New York: Academic Press, 63 101

Pontryagin L S, V G Boltyanskii, R V Gamkrelidze, et al., 1962. The Mathematical Theory of Optimal Processes. New York

Watt K E F, 1968. Ecology and Resource Management. New York: McGraw-Hill

Wickshire K. 1977. Mathematical models for the control of pests and infectious diseases: A survey. Theor. Popul. Biol. 11: 182 238

Xie Xueshu, 1986. The principle and appliance of optimal control. Beijing: Qinghua Univ. Press, 551 $\sim 560$ (in Chinese)

Yan Jingsong, 1986. The principle and type of ecological engineering turning polluted water into a resource. Rural Eco-Enviro. 4:19 23 (in Chinese) 